\title{
Dynamic threshold voltage control for dual supply voltage scheme on PD-SOI
}

\author{
Masaaki Iijima $^{1}$, Kenji Hamada ${ }^{1}$, Masayuki Kitamura ${ }^{1}$, \\ Masahiro Numa ${ }^{1 \mathrm{a})}$, Akira Tada ${ }^{2}$, and Takashi Ipposhi ${ }^{2}$ \\ ${ }^{1}$ Graduate school of science and technology, Kobe University \\ 1-1, Rokkodai, Nada, Kobe, Hyogo 657-8501, Japan \\ ${ }^{2}$ Renesas Technology Corporation \\ 4-1, Mizuhara, Itami, Hyogo 664-0005, Japan \\ a)numa@kobe-u.ac.jp
}

\begin{abstract}
The dual supply voltage (dual- $V_{\mathrm{DD}}$ ) scheme reduces the active power consumption without performance degradation by using two power supply rails. However, an increase in the delay due to the scaled-down supply voltage has made assigning the lower supply voltage $\left(V_{\mathrm{DDL}}\right)$ more difficult in the conventional dual- $V_{\mathrm{DD}}$ scheme. We propose a technique for the dual- $V_{\mathrm{DD}}$ scheme employing the Active Body-biasing Control (ABC) on PD-SOI, which increases the number of $V_{\mathrm{DDL}}$-cells owing to lowered threshold voltage. Simulation results have shown our approach effectively reduces the power consumption even at low voltage operation.
\end{abstract}

Keywords: low power, active body-bias, PD-SOI, dual- $V_{\mathrm{DD}}$

Classification: Integrated circuits

\section{References}

[1] K. Usami and M. Horowitz, "Clustered voltage scaling technique for lowpower design," Proc. of International Symposium on Low Power Design, pp. 3-8, April 1995.

[2] Y. Hirano, T. Matsumoto, S. Maeda, T. Iwamatsu, T. Kunikiyo, K. Nii, K. Yamamoto, Y. Yamaguchi, T. Ipposhi, S. Maegawa, and M. Inuishi, "Impact of $0.10 \mu \mathrm{m}$ SOI CMOS with body-tied hybrid trench isolation structure to break through the scaling crisis of silicon technology," IEEE IEDM Tech. Dig., pp. 467-470, Dec. 2000.

[3] K. Fukuoka, M. Iijima, K. Hamada, M. Numa, and A. Tada, "A novel layout approach using dual supply voltage technique on body-tied PD-SOI," Proc. of 14th International Workshop on Power and Timing Modeling, Optimization and Simulation, pp. 423-432, Sept. 2004.

[4] M. Hamada, M. Takahashi, H. Arakida, A. Chiba, T. Terazawa, T. Ishikawa, M. Kanazawa, M. Igarashi, and K. Usami, "A top-down low power design technique using clustered voltage scaling with variable supply-voltage scheme," Proc. of IEEE Custom Integrated Circuits Conf., pp. 495-498, May 1998.

[5] S. Yang, "Logic synthesis and optimization benchmarks user guide version 3.0," MCNC, Jan. 1991. 


\section{Introduction}

A drastically increased power consumption in LSIs in accordance with aggressively scaled-down CMOS technologies is becoming one of the most critical design issues especially for battery-operated devices due to severe power constraints. Lowering the supply voltage $\left(V_{\mathrm{DD}}\right)$ has been an effective approach in order to pursue low power operation since the dynamic power consumption drops in proportion to the square of $V_{\mathrm{DD}}$. The lowered supply voltage also reduces the leakage power consumption derived from the sub-threshold and gate leakage current during the standby mode. The dual- $V_{\mathrm{DD}}$ scheme [1], providing the normal supply voltage $\left(V_{\mathrm{DDH}}\right)$ for the gates on critical paths and the lower supply voltage $\left(V_{\mathrm{DDL}}\right)$ for others, allows low power operation without performance degradation. Simply scaling the supply voltage down to sub-1V, however, degrades the gate delay exponentially, which results in the difficulty in assigning the lower supply voltage. Hence, the conventional dual- $V_{\mathrm{DD}}$ scheme becomes less effective at low voltage operation. Then, we address a novel low power technique for dual- $V_{\mathrm{DD}}$ employing Active Bodybiasing Control (ABC) on PD-SOI, which aims at allocating the $V_{\mathrm{DDL}}$ adequately even at low voltage operation.

\section{Adapting PD-SOI to dual- $V_{\mathrm{DD}}$ scheme}

The main difference of a layout style in the dual- $V_{\mathrm{DD}}$ scheme between the bulk and PD-SOI process is a freedom of cell placement as shown in Fig. 1. For applying the dual- $V_{\mathrm{DD}}$ with the bulk process, designers need to separate cells in $V_{\mathrm{DDH}}$ and $V_{\mathrm{DDL}}$ rows. This is because the n-well which corresponds to the body of pMOS should be isolated between $V_{\mathrm{DDH}}$-cells and $V_{\mathrm{DDL}}$-cells, which causes quite a large area penalty. Hence, only the cells provided the same supply voltage can be placed in a row as shown in Fig. 1 (a), since standard cells have a power supply rail, either $V_{\mathrm{DDH}}$ or $V_{\mathrm{DDL}}$, and a ground rail. In this layout approach, wire lengths between $V_{\mathrm{DDH}}-\mathrm{cells}$ and $V_{\mathrm{DDL}}$-cells tend to be long. In addition, the freedom of cell placement is suppressed due to this constraint compared to the layout style with the single supply voltage.

On the other hand, the PD-SOI process, the body of which is separated by trench isolation [2], allows the almost same layout style with the single supply voltage [3]. In Fig. 1 (b), each cell has two supply voltage rails. Both

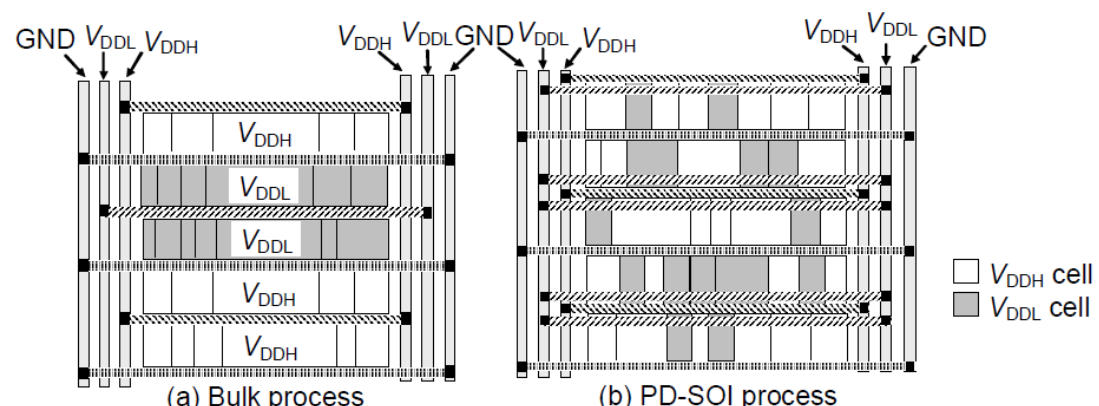

(a) Bulk process

(b) PD-SOI process

Fig. 1. Layout of the dual- $V_{\mathrm{DD}}$ 
$V_{\mathrm{DDH}}$-cells and $V_{\mathrm{DDL}}$-cells are placed in a row without any space between cells, which is different from the layout style with the bulk process. Namely, the advantage of employing PD-SOI for dual- $V_{\mathrm{DD}}$ scheme is an enhanced freedom of cell placement compared to the bulk process. We regard this dual- $V_{\mathrm{DD}}$ scheme with PD-SOI process [3] as a conventional approach, and aim for the power saving effect even at low voltage operation.

\section{Dynamic threshold voltage control}

In this section, we propose a novel low power technique by using the dynamic threshold voltage $\left(V_{\mathrm{th}}\right)$ control with Active Body-biasing Control (ABC), which is effective even at low supply voltage scaled down to sub-1V. First we describe a way of bias control, and then show a bias control circuit in detail.

Our concept includes the following three points:

i) Increase in the number of $V_{\mathrm{DDL}}$-cells.

ii) Leakage power reduction by the reverse body-bias.

iii) Using only two supply voltages $\left(V_{\mathrm{DDH}}, V_{\mathrm{DDL}}\right)$ for the body-bias control.

In the dual- $V_{\mathrm{DD}}$ scheme, the power saving effect which is actually dependent upon a trade-off between the value of $V_{\mathrm{DDL}}$ and the number of $V_{\mathrm{DDL}}$-cells reportedly becomes the maximum when $V_{\mathrm{DDL}}=0.6 \sim 0.7 V_{\mathrm{DDH}}[4]$. Under this condition, we aim at assigning a larger number of $V_{\mathrm{DDL}}$-cells owing to the lowered threshold voltage by the active body-bias.

We show a bias control circuit in Fig. 2. In the active mode $(\mathrm{EN}=1)$, all transistors in logic circuits are provided the forward body-bias. The body voltages of nMOSs are set to $V_{\mathrm{DDL}}$, and those of pMOSs in $V_{\mathrm{DDH}}$-cells and $V_{\mathrm{DDL}}$-cells are tied to $V_{\mathrm{DDL}}$ and the ground, respectively. Thus, the forward body-bias of " $V_{\mathrm{DDL}}$ " is assigned to all nMOSs and pMOSs in $V_{\mathrm{DDL}}$-cells, and that of " $V_{\mathrm{DDH}}-V_{\mathrm{DDL}}$ " is provided to pMOSs in $V_{\mathrm{DDH}}-\mathrm{cells}$. As a result,

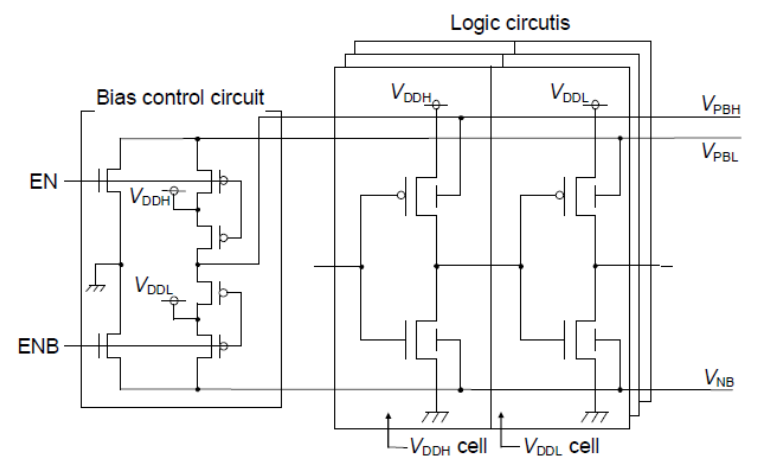

Bias condition $\left(V_{D D H}=0.8 \mathrm{~V}, V_{D D L}=0.5 \mathrm{~V}\right)$

\begin{tabular}{c|c|cc|cc}
\multicolumn{2}{c|}{ Bias condition $\left(V_{\mathrm{DDH}}=0.8 \mathrm{~V}, V_{\mathrm{DDL}}=0.5 \mathrm{~V}\right)$} \\
\hline \multicolumn{2}{c|}{ Transistor } & \multicolumn{2}{c|}{$\mathrm{nMOS}$} & \multicolumn{2}{c}{$\mathrm{pMOS}$} \\
\hline \multicolumn{2}{c|}{ Supply voltage } & $V_{\mathrm{DDL}}$ & $V_{\mathrm{DDH}}$ & $V_{\mathrm{DDL}}$ & $V_{\mathrm{DDH}}$ \\
\hline \multirow{2}{*}{ Body-bias [V] } & Active mode & 0.5 & 0.5 & 0 & 0.5 \\
& Standby mode & 0 & 0 & 0.8 & 0.8 \\
\hline
\end{tabular}


a critical path delay can be shortened in comparison with the conventional $V_{\text {th}}$-fixed approach [3]. Hence, the number of $V_{\mathrm{DDL}}$-cells increases as long as the timing constraint for the single supply voltage is satisfied, which therefore reduces the power consumption.

In addition, in the standby mode $(\mathrm{EN}=0)$, the body voltages of nMOSs and pMOSs are set to the ground and $V_{\mathrm{DDH}}$, respectively. Thus, the reverse body-bias of " $V_{\mathrm{DDH}}-V_{\mathrm{DDL}}$ " is provided to pMOSs in $V_{\mathrm{DDL}}$-cells, while all nMOSs and pMOSs in $V_{\mathrm{DDH}}$-cells are in zero body-bias condition. The higher threshold voltage of pMOSs in $V_{\mathrm{DDL}}$-cells contributes to leakage power reduction. Moreover, our approach also has an advantage of making use of two power supply rails in the bias control circuit. In general, the bias control circuit requires additional supply voltages for nMOSs and pMOSs. Thus, the total number of supply voltages becomes three or four, in the case of the single supply voltage or the dual- $V_{\mathrm{DD}}$ scheme. Then, our approach employs only two supply voltages $\left(V_{\mathrm{DDH}}, V_{\mathrm{DDL}}\right)$ for body-bias control, which saves power resources.

\section{Circuit simulation and results}

In order to examine our proposed approach, first, we have designed the cell layout including the body-bias control lines with two supply voltages $\left(V_{\mathrm{DDH}}\right.$, $\left.V_{\mathrm{DDL}}\right)$. Each cell has two supply voltages $\left(V_{\mathrm{DDH}}, V_{\mathrm{DDL}}\right)$ and three body-bias control lines for nMOS and pMOS $\left(V_{\mathrm{NB}}, V_{\mathrm{PBH}}, V_{\mathrm{PBL}}\right)$ as shown in Fig. 2. Thus, an area overhead of our approach derived from the ratio of cell heights relative to the single supply voltage and the conventional dual- $V_{\mathrm{DD}}$ is $44 \%$ and $18 \%$, respectively. The proposed cell height is higher than the others due to multiple power rails. Most of area overhead depends on the ratio of cell heights. Although we have designed the cell layout using up to the metal2 layer, it would be effective for cell area reduction that we take account of multiple metal layers for power rails.

Next, we have applied our proposed approach to MCNC benchmark circuits [5] with $0.18 \mu \mathrm{m}$ PD-SOI technology. Then, we have performed HSPICE simulation with the RC parameters extracted from the layout. Here, the supply voltages have been set to $V_{\mathrm{DDH}}=0.8 \mathrm{~V}$, and $V_{\mathrm{DDL}}=0.5 \mathrm{~V}$. In order to avoid exponentially increase in PN current from the body of MOSFET to the source/drain, we have determined $V_{\mathrm{DDL}}=0.5 \mathrm{~V}$ directly connected to the body. The value of $V_{\mathrm{DDH}}$ has been decided based on the relationship that $V_{\mathrm{DDL}}=0.6 \sim 0.7 V_{\mathrm{DDH}}$ for the minimum power consumption [4]. For these supply voltages, the conditions of forward or reverse body-bias are concretely shown in Fig. 2. In the active mode, all nMOSs and pMOSs in $V_{\mathrm{DDL}}$-cells are provided the forward body-bias of $0.5 \mathrm{~V}$, and pMOSs in $V_{\mathrm{DDH}}$-cells are assigned that of $0.3 \mathrm{~V}$. Then, in the standby mode, pMOSs in $V_{\mathrm{DDL}}$-cells are provided the reverse body-bias of $0.3 \mathrm{~V}$, while others are in zero body-bias condition. The threshold voltages with MOSFETs are set to $V_{\text {th-n }}=0.34 \mathrm{~V}$, $V_{\text {th-p }}=-0.42 \mathrm{~V}$ at zero body-bias state. We have decided the transistor size in the bias control circuit to the followings that $W_{\mathrm{n}}=1.5 \mu \mathrm{m}$ for nMOSs, and 
$W_{\mathrm{p}}=3.9$ or $1.9 \mu \mathrm{m}$ for pMOSs propagating $V_{\mathrm{DDL}}$ and $V_{\mathrm{DDH}}$, respectively. We have evaluated the ratio of $V_{\mathrm{DDL}}$-cells, delay, and power consumption.

\subsection{Evaluation of $V_{\mathrm{DDL}}$-cell assignment}

In the dual- $V_{\mathrm{DD}}$ scheme, power consumption can be reduced by assigning a large number of $V_{\mathrm{DDL}}$-cells. However, simply assigning $V_{\mathrm{DDL}}$-cells has possibility that a path may violate timing constraint even if the path satisfies it before the replacement. Then, we have defined a critical path of the whole circuit as the maximum delay path, and have replaced $V_{\mathrm{DDH}}-\mathrm{cells}$ with $V_{\mathrm{DDL}^{-}}$ cells not to exceed the critical path delay. Here, we show the evaluation of $V_{\mathrm{DDL}}$-cell assignment in Fig. 3 (a), where the timing constraint is for the single supply voltage. The conventional approach which does not employ the active body-bias control results in $V_{\mathrm{DDL}}$-cell assignment of only $3 \sim 13 \%$ at such low supply voltage as $V_{\mathrm{DDL}}=0.5 \mathrm{~V}$. On the other hand, our proposed approach improves up to that of $37 \sim 51 \%$ owing to lowering the threshold voltage by the active body-bias. In addition, even the critical path delay can be shortened since all cells are in the forward body-bias state. As a result, we can replace $V_{\mathrm{DDH}}$-cells with $V_{\mathrm{DDL}}$-cells even on the critical path as long as meeting the timing constraint.

\subsection{Power saving effect}

In order to evaluate the power saving effect, we have compared our proposed approach with the single supply voltage and the conventional dual$V_{\mathrm{DD}}$ scheme. We have calculated the critical path delay and active/standby power consumption including the power of bias-control circuit, where each input has been fixed, on average for 100 random input vectors at $10 \mathrm{MHz}$ based on HSPICE simulation. The results with MCNC benchmark circuits are shown in Fig. 3 (b).

With respect to the delay, the conventional dual- $V_{\mathrm{DD}}$ scheme results in the delay penalty of $3 \%$ relative to the single supply voltage due to an increase in wire length besides a lack of $V_{\mathrm{DDL}}$-cell assignment. On the other hand, our approach conceals the delay penalty in spite of area overhead in the cell layout since the active body-bias control shortens the critical path delay quite effectively.

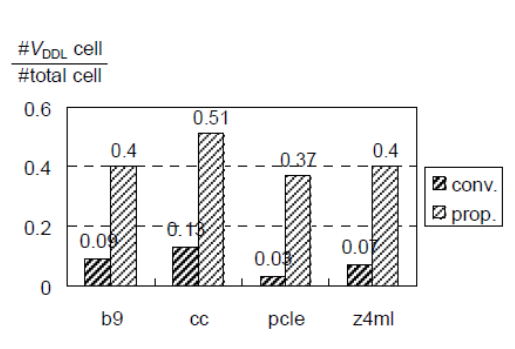

(a) Ratio of $V_{\mathrm{DDL}}$ cell

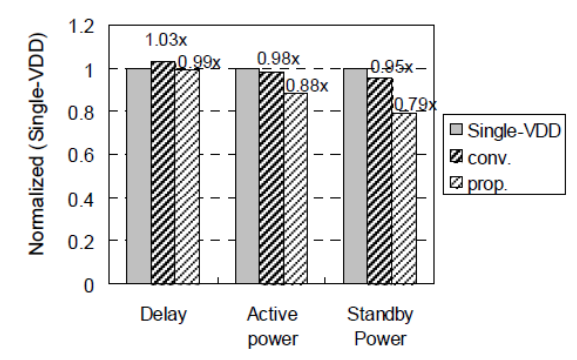

(b) Delay and power consumption

Fig. 3. Simulation results 
Next, we discuss the active power consumption. In the conventional dual$V_{\mathrm{DD}}$ scheme, a severe critical path delay constraint causes extremely lack of $V_{\mathrm{DDL}}$-cell assignment. Thus, the power saving effect is restricted to only $2 \%$. Hence, simply applying the conventional dual- $V_{\mathrm{DD}}$ scheme for low voltage operation is considered less effective any more. In our approach, however, reduces $12 \%$ of the active power consumption owing to the active body-bias control, where large number of $V_{\mathrm{DDH}}$-cells can be replaced with $V_{\mathrm{DDL}}$-cells.

Finally, we describe the results from the point of the standby power consumption. Even if all transistors are in zero body-bias condition in the conventional dual- $V_{\mathrm{DD}}$ scheme, it reduces $5 \%$ of the power consumption compared to the single supply voltage due to the lowered standby leakage power in $V_{\mathrm{DDL}}$-cells. Furthermore, our approach achieves the standby power reduction of $21 \%$ caused by the following two factors. One is the increase in $V_{\mathrm{DDL}}$-cell assignment, and the other is the higher threshold voltage of pMOSs in $V_{\mathrm{DDL}}$-cells derived from the reverse body-bias.

Therefore, although lowering the supply voltage degrades power saving effect in conventional dual- $V_{\mathrm{DD}}$ scheme, applying the active body-bias control to the dual- $V_{\mathrm{DD}}$ scheme in order to display the effect to the full enables low power operation even at low supply voltage, where the key idea is the increase in $V_{\mathrm{DDL}}$-cell assignment.

\section{Conclusion}

In this paper, the issue of conventional dual- $V_{\mathrm{DD}}$ scheme in accordance with lowering supply voltage has been picked up, and we have proposed a novel low power technique employing the active body-bias control on PD-SOI. The conventional approach suffered from the difficulty in assigning the lower supply voltage $\left(V_{\mathrm{DDL}}\right)$ since the gate delay increases exponentially as lowered supply voltage. Then, we have tried to improve the $V_{\mathrm{DDL}}$-cell assignment by dynamically lowering the threshold voltage. Simulation results with MCNC benchmark circuits based on HSPICE simulation have shown improvement in both the active and standby power consumption by $12 \%$, and $21 \%$, respectively, while maintaining the operation speed compared to the single supply voltage. 